\title{
Serum lipase should be the laboratory test of choice for suspected acute pancreatitis
}

\author{
S Hofmeyr, ${ }^{1}$ MB ChB, MMed, FCS (SA), Cert Gastroenterol (SA) Surg; C Meyer, ${ }^{2}$ MB ChB, MMed; \\ B L Warren, ${ }^{1} \mathrm{MB} \mathrm{ChB}, \mathrm{MMed}, \mathrm{FCS}(\mathrm{SA})$, FRCS \\ ${ }^{1}$ Division of Surgery, Faculty of Medicine and Health Sciences, Stellenbosch University and \\ Tygerberg Academic Hospital, Cape Town, South Africa \\ ${ }^{2}$ Division of Chemical Pathology, Faculty of Medicine and Health Sciences, Stellenbosch University and \\ National Health Laboratory Service, Tygerberg Academic Hospital, Cape Town, South Africa
}

Corresponding author: S Hofmeyr (stefhofmeyr@sun.ac.za)

Background. Serum lipase and amylase are biochemical analyses used to establish the diagnosis of acute pancreatitis (AP). Despite lipase having been shown internationally to be a more sensitive and specific test, amylase remains a popular first-line test.

Objective. To provide a local basis for the recommendation of the best first-line laboratory test, an assessment of their performance in our local setting was undertaken.

Methods. From a prospective dataset on patients with acute abdominal pain and raised serum lipase and/or amylase values, the sensitivity and specificity of serum lipase, amylase and the two in combination was calculated for the diagnosis of AP, as defined by the Atlanta criteria. Results. During the study period, 476 patients presented with acute upper or generalised abdominal pain and raised serum amylase and/or lipase values. The median age of the patients was 43 years (range 14 - 85), and 58\% were men and 42\% women. Of the patients, 322 (68\%) presented with abdominal conditions other than AP, and 154 (32\%) had AP. Ethanol abuse and gallstones accounted for 55\% and $23 \%$ of cases of AP, respectively. Lipase displayed a sensitivity of $91 \%$ for AP, against $62 \%$ for amylase. Specificity was $92 \%$ for lipase and $93 \%$ for amylase. Dual testing with lipase and amylase had a sensitivity of $93 \%$.

Conclusions. Lipase is a more sensitive test than amylase when utilising cut-off levels to diagnose AP. Lipase should replace amylase as the first-line laboratory investigation for suspected AP.

S Afr J Surg 2014;52(3):72-75. DOI:10.7196/SAJS.2003

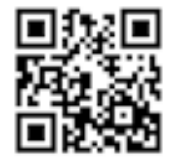

In the diagnosis of acute pancreatitis (AP), serum amylase and more recently lipase are the commonly used laboratory tests available to clinicians in South Africa (SA). Although the international literature has shown serum amylase to be a less sensitive and specific test for AP than serum lipase, ${ }^{[1-6]}$ serum amylase remains the most popular first-line investigation for suspected AP owing to the perception that the test is less expensive.

Key to the diagnosis of AP are abdominal pain consistent with AP, serum pancreatic enzyme elevation more than three times the upper limit of normal (ULN), and typical findings on cross-sectional imaging. The latter is usually contrast-enhanced computed tomography (CECT). The presence of any two is considered diagnostic. ${ }^{[7]}$ Of the two non-clinical criteria for a diagnosis of AP, in terms of convenience and cost-containment it would be logical to favour the laboratory test ahead of the computed tomography (CT) scan, provided that reliance can be placed on the accuracy of serum lipase or amylase levels. Use of an inferior test may lead to a less accurate and timely diagnosis of AP or other conditions that may mimic it, as well as unnecessary reliance on other investigations to counteract the lack of sensitivity and specificity of the less accurate test.
The proposed merits of serum lipase over amylase were not analysed in the three SA publications on AP. ${ }^{[8-10]}$ Establishing whether lipase is a better test in our population would provide local evidence that serum lipase should be used instead of amylase as a first-line test for suspected AP.

\section{Objective}

We aimed to investigate the relative performance of serum lipase and amylase at our institution, in terms of both specificity for AP and sensitivity for the diagnosis of AP at the proposed threshold of three times the ULN. The data were also analysed to determine whether dual testing of serum lipase and amylase had any benefit over serum lipase as a 'stand-alone' test.

\section{Methods}

From January 2010 to July 2012 at Tygerberg Academic Hospital, Cape Town, South Africa, data were collected prospectively on all patients presenting to the surgical admissions unit with acute generalised or upper abdominal pain and initial raised serum lipase and/or amylase values.

Inclusion criteria were acute generalised or upper abdominal pain of less than 5 days' duration, with or without radiation to 
the back, and a raised serum lipase and/ or amylase value. Patients with a history of chronic abdominal pain were also included if they had an acute exacerbation requiring emergency admission, provided the diagnosis was not chronic pancreatitis. Patients were excluded if they were under 13 years of age or had a history of recent penetrating or blunt abdominal trauma or incomplete/inconclusive clinical records. Clinical, endoscopic, operative, laboratory and imaging data were reviewed to confirm the final diagnosis for each case. Data collected were age, gender, value of raised serum amylase and lipase, diagnosis and method of diagnosis.

AP was diagnosed if at least two of the three criteria of the revised Atlanta classification $^{[7]}$ were met. All patients who presented with pain and findings typical of AP on clinical examination, but a serum lipase and amylase elevation of less than three times the ULN, had CECT or laparotomy to confirm the diagnosis. CECT was also used to exclude other conditions presenting with enzyme elevations of more than three times the ULN if the clinical findings were atypical for AP.

In cases where one of the serum markers was raised above the ULN but not the other, the value of the other marker was included as well. Conduct of the study was approved by the Health Research Ethics Committee of the Faculty of Medicine and Health Sciences at Stellenbosch University (reference N08/01/008).

The data were entered into an electronic spreadsheet. Descriptive statistical analysis was performed and results were expressed as percentages, medians and ranges. Sensitivity and specificity for both enzymes for the diagnosis of AP were calculated using the cut-off of three times the ULN.

The samples were analysed in the Tygerberg Hospital National Health Laboratory Service (NHLS) laboratory, which is accredited by the South African National Accreditation System and participates in external quality control. The analytes were measured using a Siemens Advia 1800 system. The normal range for serum lipase was $0-60 \mathrm{U} / \mathrm{L}$ and that for serum amylase $28-110 \mathrm{U} / \mathrm{L}$.

\section{Results}

During the study period, 760 patients presented with abdominal pain and a raised serum lipase and/or amylase value. Of these, 476 met the inclusion criteria and were included in the final analysis. The median age of these patients was 43 years (range $14-85$ ), and $58 \%$ were male and $42 \%$ female.

Of the 476 patients, 322 (68\%) presented with abdominal conditions other than AP. There were 25 extrapancreatic diagnoses, the most common being gastritis/non-perforated peptic ulcer, perforated gastric or duodenal ulcer, intestinal obstruction, nonspecific abdominal pain, appendicitis and abdominal tuberculosis. In 154 patients, the final diagnosis was AP. The cause of the AP was ethanol abuse in 55\% and gallstones in $23 \%$. Causes of AP, with corresponding serum enzyme values, are summarised in Table 1.

Table 2 shows the distribution of pancreatic enzyme elevations above and below the cut-off level. Above three times the ULN, specificity for AP was similar, with lipase and amylase being 92\% and 93\% specific for AP, respectively. The most striking difference between the enzymes was in sensitivity for AP. Above the threshold level of three times the ULN, lipase displayed a superior sensitivity of $91 \%$ for AP as opposed to $62 \%$ for amylase. At the cut-off level of three times the ULN, amylase as a single test would not have been diagnostic in $38 \%$ of cases of AP.

The sensitivity of amylase and lipase was calculated separately for ethanoland gallstone-induced AP. Lipase was considerably more sensitive than amylase above the cut-off level for ethanol-induced AP $(91 \%$ v. $52 \%)$, and was also more sensitive for gallstone-induced AP (97\% v. $80 \%$ ) (Table 3).

The data were analysed to determine whether dual testing with both enzymes would lead to superior sensitivity for AP
Table 1. Causes of AP

\begin{tabular}{llll}
\hline Cause & $\boldsymbol{n}(\%)$ & $\begin{array}{l}\text { Lipase (U/L), } \\
\text { median }(\text { range })\end{array}$ & $\begin{array}{l}\text { Amylase (U/L), } \\
\text { median (range) }\end{array}$ \\
\hline Ethanol & $85(55.2)$ & $364(70-2964)$ & $331.5(41-1972)$ \\
Gallstones & $35(22.7)$ & $1114(111-10000)$ & $874.5(158-5292)$ \\
Idiopathic & $17(11.0)$ & $670.5(30-2843$ & $487.5(133-3336)$ \\
Other & $17(11.0)$ & $636(59-5135)$ & $973(147-2182)$ \\
Total & 154 & $567(30-10000)$ & $402(41-5292)$ \\
AP = acute pancreatitis. & &
\end{tabular}

Table 2. Specificity/sensitivity of lipase and amylase for AP at cut-off level

\begin{tabular}{lll}
\hline & Acute pancreatitis, $\boldsymbol{n}$ & Extrapancreatic conditions, $\boldsymbol{n}$ \\
\hline Serum lipase & & 25 \\
$>3 \times$ ULN & 140 & 297 \\
$<3 \times$ ULN & 14 & \\
Serum lipase specificity for AP above threshold $=92 \%$ \\
Serum lipase sensitivity for AP above threshold $=91 \%$ \\
Serum amylase \\
$\begin{array}{l}>3 \times \text { ULN } \\
<3 \times \text { ULN }\end{array}$ \\
$\begin{array}{l}\text { Serum amylase specificity for AP above threshold }=93 \% \\
\text { Serum amylase sensitivity for AP above threshold }=62 \%\end{array}$ \\
AP $=$ acute pancreatitis; ULN = upper limit of normal.
\end{tabular}


over lipase alone. Table 4 sets out the distribution of AP cases that presented with an elevation in either lipase or amylase above three times the ULN. This shows that the sensitivity of dual testing above the threshold level is $93 \%$, which is only marginally better than the $91 \%$ achieved by lipase as a single test.

\section{Discussion}

The presenting symptoms of AP (epigastric or generalised abdominal pain, nausea and vomiting) are not exclusive to this disease but are shared by other abdominal emergencies, many requiring surgical intervention, such as peptic ulcer perforation and intestinal obstruction. The attending clinician can therefore not make a reliable diagnosis without the use of selected laboratory and imaging investigations. Serum lipase and amylase measurement are both commonly used in patients presenting with clinical features suspicious of AP, as a means of either confirming or excluding the diagnosis of AP. Use of these enzymes in this context is, however, subject to an understanding of their imperfections.

The first of these is the lack of specificity of these enzymes for AP, which relates to the ability to exclude AP in patients presenting with acute abdominal pain. It is well known that other intra-abdominal disorders, such as peptic ulcer perforation, mesenteric ischaemia and intestinal obstruction, may present with raised serum lipase/amylase values. ${ }^{[3,5,6,11]}$ These elevations are usually mild (typically between one and two times the ULN). Although these extrapancreatic elevations may on occasion be very high, many authors have suggested that a cut-off level of three times the ULN be used, thereby increasing the specificity of the enzymes for AP. ${ }^{[1,3,11]}$ These data show that, in line with other published data, ${ }^{[5-7]}$ lipase and amylase are both very specific laboratory tests for the diagnosis of AP when the suggested cut-off level is used.

The more major imperfection is the lack of sensitivity of serum enzyme elevation to identify all those who have AP. Published experience has shown that AP is less likely to present with normal serum lipase than serum amylase values, ${ }^{[5,6,11-13]}$ and this becomes more common with late

Table 3. Sensitivity of lipase and amylase for AP caused by ethanol and gallstones

\begin{tabular}{|c|c|c|}
\hline & Lipase, $n$ & Amylase, $n$ \\
\hline \multicolumn{3}{|c|}{ Ethanol-induced AP $(N=85)$} \\
\hline$>3 \times \mathrm{ULN}$ & 77 & 44 \\
\hline$<3 \times \mathrm{ULN}$ & 8 & 41 \\
\hline \multicolumn{3}{|c|}{ Serum lipase sensitivity for ethanol-induced AP above threshold $=91 \%$} \\
\hline \multicolumn{3}{|c|}{ Serum amylase sensitivity for ethanol-induced AP above threshold $=52 \%$} \\
\hline \multicolumn{3}{|c|}{ Gallstone-induced AP $(N=35)$} \\
\hline$>3 \times \mathrm{ULN}$ & 34 & 28 \\
\hline$<3 \times \mathrm{ULN}$ & 1 & 7 \\
\hline \multicolumn{3}{|c|}{ Serum lipase sensitivity for gallstone-induced AP above threshold $=97 \%$} \\
\hline \multicolumn{3}{|c|}{ Serum amylase sensitivity for gallstone-induced AP above threshold $=80 \%$} \\
\hline $\mathrm{AP}=$ acute pancre & rmal. & \\
\hline
\end{tabular}

Table 4. Sensitivity of dual enzyme testing

\begin{tabular}{lll}
\hline AP & Lipase and/or amylase, $\boldsymbol{n}$ & Lipase alone, $\boldsymbol{n}$ \\
\hline$>3 \times$ ULN & 143 & 140 \\
$<3 \times$ ULN & 11 & 14 \\
Dual testing sensitivity for AP above threshold $=93 \%$ & \\
Serum lipase sensitivity for AP above threshold $=91 \%$ & \\
AP = acute pancreatitis; ULN $=$ upper limit of normal.
\end{tabular}

presentations, when amylase levels tend to return to normal. Lipase has been shown to remain elevated longer than amylase after the onset of AP. ${ }^{[6]}$ Our data show a considerable difference in sensitivity between the two enzymes when a cutoff level is utilised to diagnose AP. Only $62 \%$ of our patients with AP presented with a serum amylase elevation more than three times the ULN, implying that as a single test, amylase failed to satisfy the proposed diagnostic criteria in $38 \%(n=58)$ of AP patients. To avoid an unnecessary laparotomy, these patients require a CT scan to confirm the diagnosis. Lipase, on the other hand, had a sensitivity of $91 \%$ at the cut-off level, demonstrating its superiority in this regard, which should translate into significantly fewer diagnostic CT scans.

These findings should be interpreted within the context of the aetiology of AP in this study. In comparison with developed countries, where gallstones may play a larger causative role, ${ }^{[14]}$ alcohol was by far the most common cause in this study. As ethanol-induced pancreatitis presents with lower serum enzyme elevations compared with gallstone pancreatitis, the sensitivity of these enzymes for AP will be negatively affected, especially when a cut-off level of three times the ULN is used.

The data showed that dual testing of lipase and amylase produced a sensitivity of $93 \%$ for AP at three times the ULN, which is an improvement of only $2 \%$ over lipase as a single test, a marginal gain achieved at double the cost. This finding is consistent with recent publications that concluded that testing of lipase alone is preferable to testing of amylase alone or both enzymes, and that this strategy may result in cost savings. ${ }^{[15,16]}$ The NHLS cost is R38 for either test, while the typical cost at a laboratory servicing the private health sector is R60 each. ${ }^{[17,18]}$

\section{Conclusion}

We have shown that serum lipase is a more sensitive test and satisfies the diagnostic criteria for AP more readily than amylase. 
Dual testing in our setting adds little benefit compared with lipase as a 'stand-alone' test. The superior diagnostic performance of serum lipase has the potential to result in more efficient patient care and cost savings owing to less reliance on CECT to investigate patients presenting with acute abdominal pain. It is therefore recommended that serum lipase should supplant amylase as the first-line laboratory investigation for suspected AP.

\section{REFERENCES}

1. Yadav D, Agarwal N, Pitchumoni CS. A critical evaluation of laboratory tests in vacute pancreatitis. Am J Gastroenterol 2002;97(6):1309-1318. [http://dx.doi.org/1 0.1111/j.1572-0241.2002.05766.]

2. Steinberg WM, Goldstein SS, Davis ND, Shamma’a J, Anderson, K. Diagnostic assays in AP: A study of sensitivity and specificity. Ann Intern Med 1985;102(5):576580. [http://dx.doi.org/10.7326/0003-4819-102-5-576]

3. Clavien PA, Burgan S, Moossa AR. Serum enzymes and other laboratory tests in acute pancreatitis. Br J Surg 1989;76(12):1234-1243. [http://dx.doi.org/10.1002/ bjs.1800761205]

4. Chase CW, Barker DE, Russell WL, Burns RP. Serum amylase and lipase in the evaluation of acute abdominal pain. Am Surg 1996;62(12):1028-1033.

5. Keim V, Teich N, Fiedler F, Hartig W, Thiele G, Mossner J. A comparison of lipase and amylase in the diagnosis of acute pancreatitis in patients with abdominal pain. Pancreas 1998;16(1):45-49. [http://dx.doi.org/10.1097/00006676-199801000-00008]

6. Smith RC, Southwell-Keely J, Chesher D. Should serum pancreatic lipase replace serum amylase as a biomarker of acute pancreatitis? Aust N Z J Surg 2005;75(6):399404. [http://dx.doi.org/10.1111/j.1445-2197.2005.03391.x]
7. Banks PA, Bollen TL, Dervenis C, et al. Classification of acute pancreatitis - 2012: Revision of the Atlanta classification and definitions by international consensus. Gut 2013;62(1):102-114. [http://dx.doi.org/10.1136/gutjnl-2012-302779]

8. Funnell, IC, Bornman PC, Weakly SP, Terblanche J, Marks IN. Obesity: An important prognostic factor in acute pancreatitis. Br J Surg 1993;80(4):484-486. [http://dx.doi.org/10.1002/bjs.1800800426]

9. Anderson F, Thomson SR, Clarke DL, Loots E. Acute pancreatitis: Demographics, aetiological factors and outcomes in a regional hospital in South Africa. S Afr J Surg 2008;46(3):83-86.

10. John KD, Segal I, Hassan H, Levy RD, Amin M. Acute pancreatitis in Sowetan Africans: A disease with high mortality and morbidity. Int J Pancreatol 1997;21(2):149-155.

11. Gumaste VV, Roditis N, Mehta D, Dave PB. Serum lipase levels in nonpancreatic abdominal pain versus acute pancreatitis. Am J Gastroenterol 1993;88(12):20512055.

12. Clavien PA, Robert J, Meyer P, et al. Acute pancreatitis and normoamylasaemia: Not an uncommon combination. Ann Surg 1989;210(5):614-620. [http://dx.doi. org/10.1097/00000658-198911000-00008]

13. Frank B, Gottlieb K. Amylase normal, lipase elevated: Is it pancreatitis? Am J Gastroenterol 1999;94(2):463-469. [http://dx.doi.org/10.1111/j.15720241.1999.878_g.x]

14. Frossard J, Steer ML, Pastor CM. Acute pancreatitis. Lancet 2008;371(9607):143152. [http://dx.doi.org/10.1016/s0140-6736(08)60107-5]

15. Sutton PA, Humes DJ, Purcell G, et al. The role of routine assays of serum amylase and lipase for the diagnosis of acute abdominal pain. Ann R Coll Surg Engl 2009;91(5):381-384. [http://dx.doi.org/10.1308/003588409x392135]

16. Gomez D, Addison A, De Rosa A, et al. Retrospective study of patients with acute pancreatitis: Is serum amylase still required? BMJ Open 2012;2(5):e001471. [http:// dx.doi.org/10.1136/bmjopen-2012-001471]

17. Pathcare pathology group. www.pathcare.co.za/Pathcare.php? text=Fees\&Id=0\&im age=FeesEnquiries.jpg (accessed 29 August 2013).

18. National Health Laboratory Service. State pricing catalogue 2011/2012. www.doh. gov.za/docs/programmes/2012/appendixM.pdf (accessed 29 August 2013). 